\title{
Generation mechanism of Z-mode waves in the equatorial plasmasphere
}

\author{
Y. Nishimura ${ }^{1}$, T. Ono ${ }^{1}$, M. Iizima ${ }^{1}$, A. Shinbori ${ }^{2}$, and A. Kumamoto ${ }^{1}$ \\ ${ }^{1}$ Department of Geophysics, Tohoku University, Sendai 980-8578, Japan \\ ${ }^{2}$ Solar-Terrestrial Environment Laboratory, Nagoya University, Aichi 464-8601, Japan \\ (Received February 20, 2007; Revised June 10, 2007; Accepted August 5, 2007; Online published September 28, 2007)
}

\begin{abstract}
In order to clarify the generation mechanism of Z-mode waves observed in the equatorial plasmasphere, the growth rate of Z-mode electromagnetic waves has been calculated under the higher-order cyclotron interaction process. Z-mode waves can interact with some tens of $\mathrm{keV}$ electrons with large pitch angles even in the dense cold background, and the amplitude is consistent with the Akebono plasma wave measurements. UHR and whistler mode waves are also excited by the same electron distribution, and this is also consistent with observations. The origin of these energetic electrons are identified as the ring current electrons injected into the plasmasphere by the intense large-scale electric field during geomagnetic storms, accelerated perpendicular to the ambient magnetic field and confined around the geomagnetic equator conserving the first and second adiabatic invariants. Since the intensity of Z-mode and UHR waves is associated with the development and decay of the ring current, ring current particles are most possible candidate for the free energy source of these waves.
\end{abstract}

Key words: UHR, Z-mode, plasma wave, cyclotron resonance, magnetosphere, magnetic equator.

\section{Introduction}

UHR (slow Z-mode) waves are identified as weak continuous emissions and measured in the wide region of the magnetosphere and plasmasphere (Mosier et al., 1973; Christiansen et al., 1978). Since these waves are observed continuously and the frequency depends on the plasma frequency, these emissions have been used to estimate local electron density (Bauer and Stone, 1968). Z-mode electromagnetic emissions (fast Z-mode) have been reported in the auroral magnetosphere, following to R-X or L-O mode auroral kilometric radiations (Calvert, 1966) and in ionospheric sounding echoes (Eckersley, 1933). Although it is difficult to distinguish Z-mode waves from UHR waves in the high$\omega_{p} / \Omega_{e}$ region of the magnetosphere, natural Z-mode waves have been identified in the topside ionosphere in the region of $\omega_{p} \Omega_{e}>1$ (Horita and James, 2004).

UHR and Z-mode waves frequently enhance in the geomagnetic equatorial region of the plasmasphere, which has been called the equatorial enhancement of the plasma wave turbulence (EPWAT) (Oya et al., 1990, 1991). Although the Akebono satellite has not performed particle measurements around the equator, it has been considered that these plasma wave enhancements are associated with energetic particles injected into the inner plasmasphere during geomagnetic storms, since the intensity of these waves increases during geomagnetic active periods (Oya et al., 1991; Nishimura et al., 2006).

Since UHR waves can interact with thermal electrons, it has been thought that these emissions are related to commonly existing thermal plasma or the radiation belt parti-

Copyright (c) The Society of Geomagnetism and Earth, Planetary and Space Sciences (SGEPSS); The Seismological Society of Japan; The Volcanological Society of Japan; The Geodetic Society of Japan; The Japanese Society for Planetary Sciences; TERRAPUB. cles in the magnetosphere through the coherent Cerenkov process (or inverse Landau damping) (Harvey, 1968; Gregory, 1969). Z-mode waves, however, cannot resonate with thermal electrons through Landau-type interactions, because their phase velocities are larger than the speed of light in vacuum. Therefore, it has been thought that, since Z-mode waves are connected with electrostatic modes, Zmode waves are generated as the result of the linear mode conversion from UHR mode waves (Bauer and Stone, 1968; Jones, 1980; Oya, 1991).

However, the previous study suggested that the intensity of Z-mode waves was the same order or larger than that of the UHR waves (Nishimura et al., 2006). In addition, as will be shown in Fig. 1, intense Z-mode waves are sometimes detected without enhancement of UHR waves. These evidences suggest that Z-mode waves are not simply converted from electrostatic waves but generated by a direct process. Although it has been widely believed that a low plasma density background is a necessary condition to excite high-frequency electromagnetic waves directly (Melrose et al., 1984), the cyclotron resonance condition itself can be satisfied for the higher-order resonance even in the dense plasmaspheric plasma. In the present paper, we discuss how effectively Z-mode electromagnetic waves are excited directly in the equatorial region of the plasmasphere through a higher-order cyclotron resonance. Numerical calculations of wave growth rates is performed by considering energetic electrons with large pitch angles, and we will show that large growth rates can be obtained through the higher-order cyclotron resonance with Z-mode electromagnetic waves in high- $\omega_{p} / \Omega_{e}$ regions. Finally, we will propose a whole story to produce such energetic electrons, which become the energy source of EPWAT phenomena. 


\section{Basic Equations}

The Akebono satellite was launched on February 22, 1989 into a quasi-polar orbit with an inclination of $75.1^{\circ}$ with an initial perigee and apogee of $274 \mathrm{~km}$ and 10,500 $\mathrm{km}$, respectively. It continues its observations for more than 18 years, with a relatively shorter orbital period of 3.5 hours. The plasma wave and sounder (PWS) system on board the Akebono satellite measures two components of electric fields with a time resolution of 2 seconds in a frequency range between $20 \mathrm{kHz}$ and $5.2 \mathrm{MHz}$ (Oya et al., 1990). We have employed the following definition to separate UHR and Z-mode waves where $\omega_{p} / \Omega_{e}>1$ following to the definition by Oya et al. (1990). The left-handed plasma wave observed in a frequency range of $\omega_{z} \leq \omega \leq$ $\omega_{p}$ is Z-mode. The right-handed plasma wave observed in a range of $\omega_{p} \leq \omega \leq \omega_{\mathrm{UHR}}$ is UHR mode. The Z-mode waves have electromagnetic nature and resonate with energetic electrons through cyclotron resonances, while the UHR mode waves have electrostatic nature and are mainly generated by thermal electrons through Landau-type interaction processes. In general, both modes are called the $\mathrm{Z}$-mode wave, but we distinguish these modes in order to study the origin of these waves.

\subsection{Injection of energetic electrons}

The following relativistic guiding center equations is solved numerically to investigate the evolution of pitch angle, energy, as well as trajectories of energetic electrons (Roederer, 1970):

$$
\begin{aligned}
m_{0} \frac{d \mathbf{u}_{\|}}{d t}= & q\left(\mathbf{E}_{\|}-\gamma \mu \nabla_{\|} B\right) \\
\gamma \frac{d \mathbf{x}_{d}}{d t}= & \gamma \frac{\mathbf{E} \times \mathbf{B}}{B^{2}}-\frac{m_{0}}{2 q B}\left(\mathbf{u}_{\perp}^{2}+2 \mathbf{u}_{\|}^{2}\right) \frac{\nabla B \times \mathbf{B}}{B^{2}} \\
& +\frac{\gamma}{B} \frac{d}{d t}\left(\frac{\mathbf{E}_{\perp}}{\Omega_{e} / \gamma}\right)
\end{aligned}
$$

where $m_{0}$ is the rest mass, $\mathbf{u}$ is the momentum normalized by $m_{0}, \mu$ is the relativistic first magnetic invariant, $\Omega_{e}$ is the cyclotron frequency, and $\gamma$ is the Lorentz factor, respectively. This equation only includes $\mathbf{E} \times \mathbf{B}, \nabla B$, and polarization drifts, and exclude plasma wave fields, which violate the conservation of the first adiabatic invariant and lead to pitch angle and energy diffusion. The electric field is given by the statistical study of the Akebono satellite measurements (Nishimura et al., 2007) with the dipole magnetic field.

\subsection{Cyclotron resonance with energetic electrons}

In order to evaluate the possibility of the direct excitation of Z-mode waves in the equatorial plasmasphere, we have calculated linear growth rates of plasma waves under the existence of energetic electrons and dense cold electrons. The major component of particle distribution is the ambient dense cold plasma with the temperature of $1 \mathrm{eV}$ (Kutiev et al., 2002), and the minor component is hot plasma with the energy of the order of $10 \mathrm{keV}$ (Maynard et al., 1996). As the valid conditions for the linearization, we have performed the calculation under the wave magnetic component much smaller than the ambient magnetic field, and the linear growth or damping rate much smaller than wave frequencies. The real part of the wave frequency and group velocity is given by the Appleton-Hartree's dispersion relation: This is because when discussing the growth rate of Z-mode waves with the phase velocity of larger than the speed of light in vacuum, thermal effects can be safely neglected for the real part of the wave frequency. For the case of UHR waves, this assumption is valid where the wavelength is longer than 100 times of the electron gyroradius with the speed of light. The linear growth rate is calculated following to the hot plasma theory (Baldwin et al., 1969):

$$
\begin{aligned}
\omega_{i}= & \sum_{s} \frac{2 \pi^{2} \omega_{p s}^{2}}{G \omega^{2} n_{s}} \int d \mathbf{u} \sum_{n} \frac{u_{\perp} n \Omega_{e}}{\gamma k_{\perp}}\left(\alpha_{1} J_{n}^{2}\right. \\
& \left.+\alpha_{2} J_{n} J_{n+1}+\alpha_{3} J_{n+1}^{2}\right)\left(n \Omega_{e} \frac{\partial}{\partial u_{\perp}}+k_{\|} u_{\perp} \frac{\partial}{\partial u_{\|}}\right) \\
& f_{s}\left(u_{\perp}, u_{\|}\right) \cdot \delta\left(\gamma \omega-k_{\|} u_{\|}-n \Omega_{e}\right)
\end{aligned}
$$

where $n$ is the order of resonance, $J_{n}$ is the $n$-th Bessel function, $n_{s}$ is the electron density of $s$ species (cold and hot electrons). The coefficients $\alpha_{1}, \alpha_{2}, \alpha_{3}$, and $G$ are functions of the wave frequency, wavenumber, plasma frequency, cyclotron frequency, and momentum of the energetic particles as described by Lee et al. (1979). The particles which satisfy the cyclotron resonance condition can interact with plasma waves, and the gradients of distribution functions in the momentum space determine whether the wave grows or damps.

As will be shown in the next section, energetic electrons can be injected into the plasmasphere during geomagnetic storms and become a candidate for the free energy source of plasma waves. Since these electrons are accelerated perpendicularly to the ambient magnetic field by the largescale electric field, a lower energy cut-off is possible to exist in the distribution function of these electrons. In the present study, energetic electrons are assumed to have a ring distribution in the momentum space:

$$
\begin{aligned}
& f_{h}\left(u_{\|}, u_{\perp}\right)=\frac{n_{h}}{\sqrt{\pi^{3}} A_{\|} A_{\perp}^{2} c^{3}} \\
& \exp \left(-\frac{1}{A_{\perp}^{2}}\left(\frac{u_{\perp}}{c}-\frac{u_{\perp 0}}{c}\right)^{2}-\frac{1}{A_{\|}^{2}}\left(\frac{u_{\|}}{c}-\frac{u_{\| 0}}{c}\right)^{2}\right)
\end{aligned}
$$

where $A^{2}$ is the variance of a normalized momentum, which corresponds to temperature, $u_{\| 0}$ and $u_{\perp 0}$ show average momenta, and $n_{h}$ is the number density of hot plasma. Parameters are chosen as $n_{h}=1 / \mathrm{cc}, u_{\perp 0} / c=0.4 \sim 40 \mathrm{keV}$, $u_{\| 0}=0$, and $A=A_{\|}=A_{\perp}=0.1=17.5 \mathrm{keV}$, and this distribution is shown in Fig. 4. These values are estimated from the ring current measurements by Maynard et al. (1996) during disturbed periods.

The cold back ground component with the Maxwell distribution is also shown where $n_{c}=1,000 / \mathrm{cc}$, and $A=$ $1.4 \times 10^{-3}=1 \mathrm{eV}$. This plasma density of the cold background is estimated from the Akebono/PWS analysis, and the temperature is deduced from the statistical study using the Akebono satellite data (Kutiev et al., 2002). Although the cold component is dominant compared to the hot component, it is confined around the origin of the momentum space and then does not affect the wave growth or damping. 


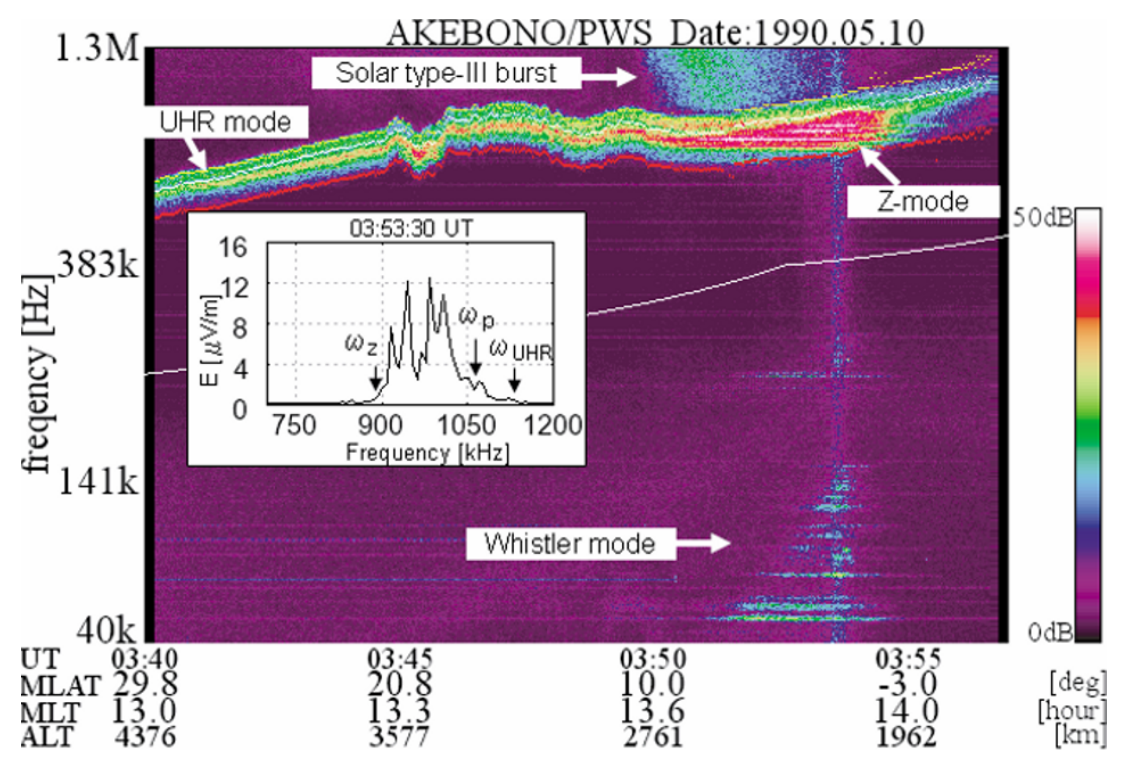

Fig. 1. Enhancement of Z-mode waves associated with whistler waves in the equatorial plasmasphere on May $10,1990 . \omega_{\mathrm{UHR}}, \omega_{p}$, and $\omega_{z}$ are shown as yellow, white and red dots, and $\Omega_{e}$ is shown as a white line. The wave intensity of 0 and $50 \mathrm{~dB}$ corresponds to $7.45[\mathrm{nV} / \mathrm{m}]$ and $24[\mu \mathrm{V} / \mathrm{m}]$, respectively. The frequency spectrum including intense Z-mode waves at 3:53:30 UT is shown in a small panel.

\section{Observations}

Figure 1 shows a dynamic spectrum observed by the Akebono/PWS on May 10, 1990 in the moderately disturbed period, including enhancement of plasma waves around the geomagnetic equator. UHR and $\mathrm{Z}$ modes are distinguished using the automatical detection of the plasma frequency (Nishimura et al., 2006), where UHR, plasma and Z-mode cut-off frequencies are indicated by yellow, white, and red dots. The Z-mode wave is enhanced just below the plasma frequency, and its intensity decreases toward the Z-mode cut-off frequency, indicating the wave propagation toward the Earth. As seen in the frequency spectrum at 3:53:30 UT, the Z-mode emission involves multi-banded discrete spectra. It suggests that a coherent wave-particle interaction process excites these Z-mode waves. The multi-banded nature is attributed to the spatial dependence of the plasma and cyclotron frequencies resulting in the change of wave frequencies satisfying the resonance condition.

The Z-mode waves enhance with the intensity of more than $10 \mu \mathrm{V} / \mathrm{m}$ in a narrow latitude range from $-2.4^{\circ}$ to $9.4^{\circ}$, while in the other latitudes, the intensity is less than $0.5 \mu \mathrm{V} / \mathrm{m}$. Thus, the $\mathrm{Z}$-mode waves are intensified up to $26 \mathrm{~dB}(=20 \times \log (10 / 0.5))$ in the equatorial region. On the other hand, UHR-mode waves do not show clear enhancement around the equatorial region.

In this event, whistler mode waves are also intensified in the equatorial region. As well as Z-mode waves, these emissions consist of the similar discrete spectra in frequency ranges of $0.15<\omega / \Omega_{e}<0.39$ and $0.68<\omega / \Omega_{e}<0.76$. These discrete spectra of Z-mode and whistler waves indicate that these waves are excited through a coherent plasma process such as the cyclotron resonance with energetic electrons.

Figure 2(a) and (b) show the temporal variation of the intensity of UHR and Z-mode waves in a geomagnetic latitude range within $\pm 40^{\circ}$ from February 1 to 15,1992 . This period includes two intense geomagnetic storms, and both the UHR and Z-mode waves have a clear tendency to enhance during the geomagnetic storms. During the main phase of the February 1-5 geomagnetic storm, both UHR and Z-mode waves intensify to $10 \mu \mathrm{V} / \mathrm{m}$ in low and midlatitude ranges, centered at the geomagnetic equatorial region. During the recovery phase, the intensity of these waves decreases with time and almost disappears on February 5. On February 8, UHR and Z-mode waves again begin to be intensified, and after the main phase of the geomagnetic storm, intensity of UHR and Z-mode waves gradually decreases, and recovers to the pre-storm level on February 11. After February 12, although weak activity of UHR and Z-mode is identified in the geomagnetic equatorial region, its intensity is about 1 order of magnitude lower than that of geomagnetic storm times. In addition, the intensity of Z-mode waves is almost equal or greater than that of UHR mode waves, especially on February 9 and 10. This suggests again that these Z-mode waves are not simply enhanced as a result of the linear mode conversion process from electrostatic waves but generated by a direct process, although we must consider the propagation effect in the observed distribution of Z-mode waves.

Next, we consider the source population of Z-mode waves in the equatorial region. During geomagnetic storms, large-scale electric field intensifies in the inner magnetosphere, and significantly contributes to the injection of energetic electrons into the near-Earth region (Wygant et al., 1998; Nishimura et al., 2007). It is expected that these strong electric fields also affect the energy and pitch angle of energetic electrons penetrated into the plasmasphere. We traced trajectories of such electrons using Eq. (2) under the storm-time electric field studied by Nishimura et al. (2007). Figure 3(a) shows trajectories of electrons with the initial position, energy and pitch angle of $X=-6.6 R_{E}$, $1 \mathrm{keV}$, and $45^{\circ}$, respectively, which are typical values of 


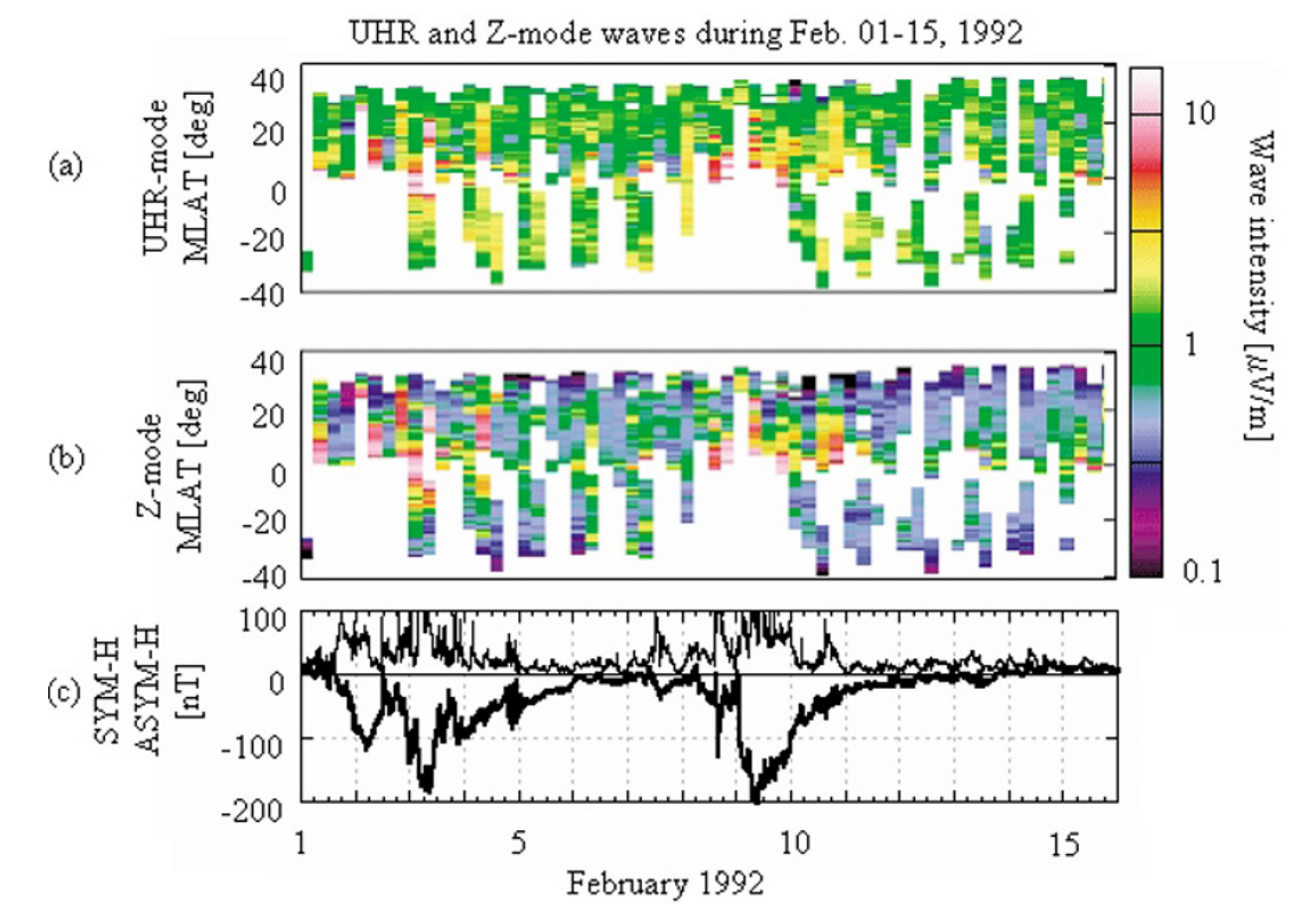

Fig. 2. Temporal variation of intensity of (a) UHR and (b) Z-mode waves, during geomagnetic storms from February 1 to 15 , 1992 . The size of each bin is 6 hours and 1 degree. The panel (c) show ASYM-H (thin black), and SYM-H (thick black) geomagnetic indices.
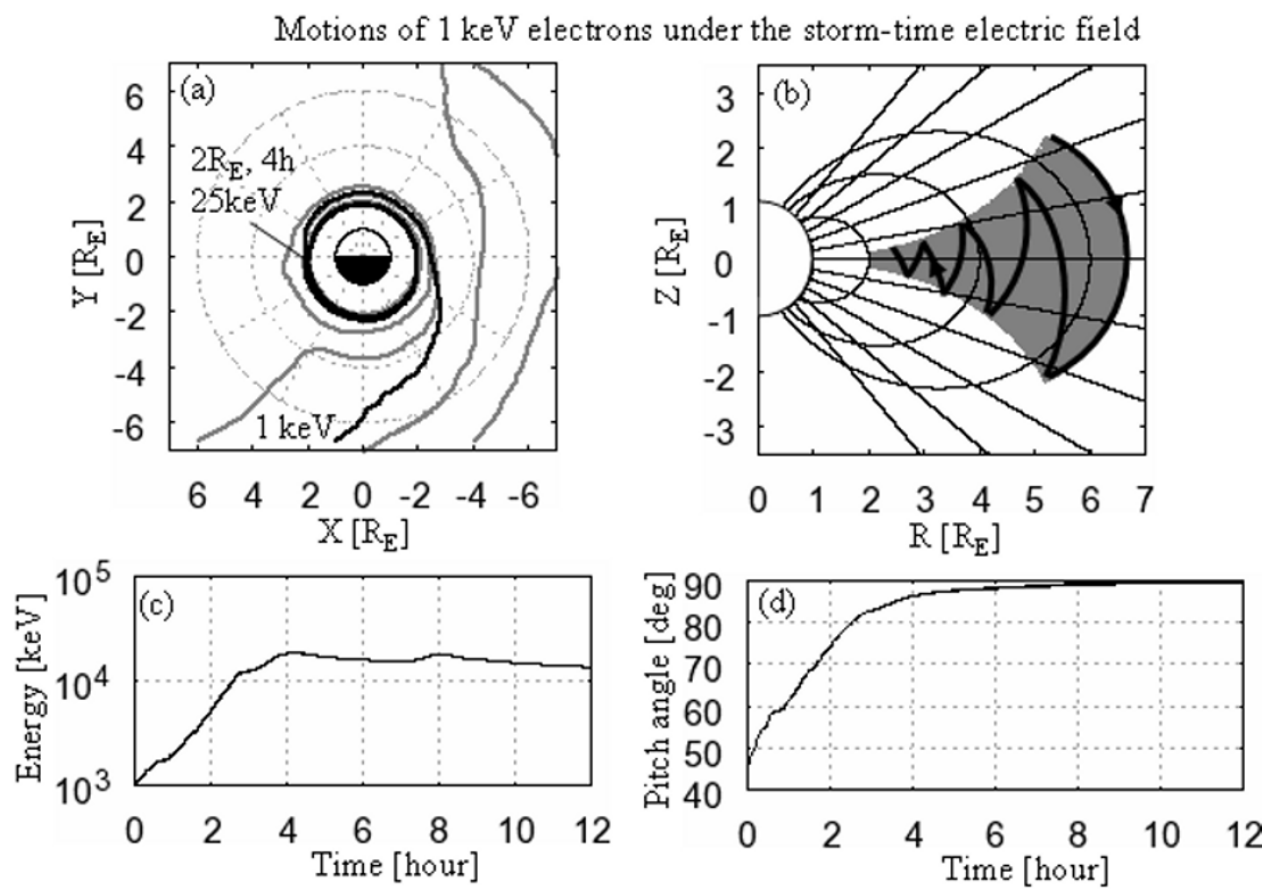

Fig. 3. The evolution of electrons with the initial position, energy, and pitch angle of $X=-6.6 R_{E}, 1 \mathrm{keV}$, and 45 degrees, respectively. The panel (a) shows trajectories in the equatorial plane. The panels from (b) to (d) show trajectory in the meridional plane, temporal variation of the energy, and pitch angle of the electron labeled by black trace in the panel (a). Electric fields are given by Akebono statistical results obtained by Nishimura et al. (2007). The black line in the panel (b) shows the schematic trail of the bouncing electron.

plasmasheet electrons. Panels from (b) to (d) show the trajectory in the meridional plane, energy, and pitch angle for the electron indicated by the black trace in the panel (a). Electrons initially at the premidnight tail region are quickly injected into $L=2$ within 4 hours, and the energy is in- creased up to $18 \mathrm{keV}$. A remarkable feature appears in its pitch angle increased to $89.6^{\circ}$ due to the conservation of the first and second adiabatic invariants, and as a result, they become equatorially mirroring electrons in the plasmasphere. Therefore, the equatorial plasmasphere is filled 
with energetic electrons with energies of the order of $10 \mathrm{keV}$ supplied from the premidnight tail during geomagnetically disturbed periods. Such equatorially mirroring energetic particles have actually been observed in the inner magnetosphere during geomagnetic storms (Burke et al., 1998; Mishin and Burke, 2005), and thus these particles are the most possible candidates for the free energy source of enhanced activities of the plasma waves confined in the equatorial region.

\section{Cyclotron Resonance with Energetic Electrons \\ 4.1 Z-mode waves}

Figure 4 represents a resonance curve (Eq. (3)) for the Z-mode with $n=3$ and ring distribution function shown in Eq. (4). Since this curve crosses the region of $\partial f / \partial u_{\perp}>0$ in the momentum space, the Z-mode wave is expected to have a positive growth rate. Since $\omega_{p} / \Omega_{e}$ is high in the plasmasphere (2.85 in Fig. 1), the Landau and first order cyclotron resonances do not occur for Z-mode waves. However, higher-order resonance conditions are satisfied, and then electromagnetic waves can be excited even in the dense cold background.

Dependence of resonance curves of Z-mode waves on wave frequency and plasma parameter is shown in Fig. 5. The wave normal angle $\theta$ is $80^{\circ}$, a representative value with a positive growth rate in Fig. 6. Curves with frequencies of $2.8 \leq \omega / \Omega_{e} \leq 2.9$ is in the region with $\partial f / \partial u_{\perp}>0$, and thus the Z-mode waves are likely to be excited with a narrow band spectrum. Resonant energies become smaller for larger wave normal angles, because the $\gamma$ factor can be smaller for the smaller contribution of the term, $k_{\|} u_{\|}$ due to the dispersion property of the Z-mode waves. It is noted that even in such low energy range, relativistic effect is essential for the resonance condition, because the curves have elliptical shape by the contribution of the $\gamma$ factor.

Figure 6 shows the growth-rate of Z-mode plasma waves using Eq. (3) in the electron distribution shown in Fig. 4. Normalized frequencies are marked beside each curve. The panel (a) shows the wave normal angle and wave frequency dependences of the wave growth rate normalized by the cyclotron frequency where $\omega_{p} / \Omega_{e}=2.9$, and $n=3$. The maximum growth rate $\omega_{i}$ is $1.1 \times 10^{-3} \Omega_{e}$ with $\omega_{r} / \Omega_{e}=$ 2.84 and $\theta=90^{\circ}$. The group velocity of this wave is $v_{g \perp} / v_{g \|}=0.47 c / 0.01 c$, which is also nearly perpendicular to the magnetic field. These waves are excited just below the ambient plasma frequency with large wave normal angles. It is because the resonant energies become smaller for larger wave normal angles. Therefore, Z-mode waves can resonate with $\mathrm{keV}$ electrons around $\omega \simeq n \Omega_{e}$. From these results, it is found that Z-mode waves effectively interact with energetic electrons with large pitch angles in the energy range of some tens of $\mathrm{keV}$ just below the plasma frequency.

For this $\omega_{p} / \Omega_{e}$, the third order resonance has the largest growth rate. For $n \leq 2$, the resonance condition is not satisfied for Z-mode waves. For $n \geq 4$, though the resonance condition can be satisfied, it requires relativistic electrons with the energy larger than $100 \mathrm{keV}$. That particle population is much less than $1-10 \mathrm{keV}$ electrons, and the growth rate is more than one order of magnitude smaller.

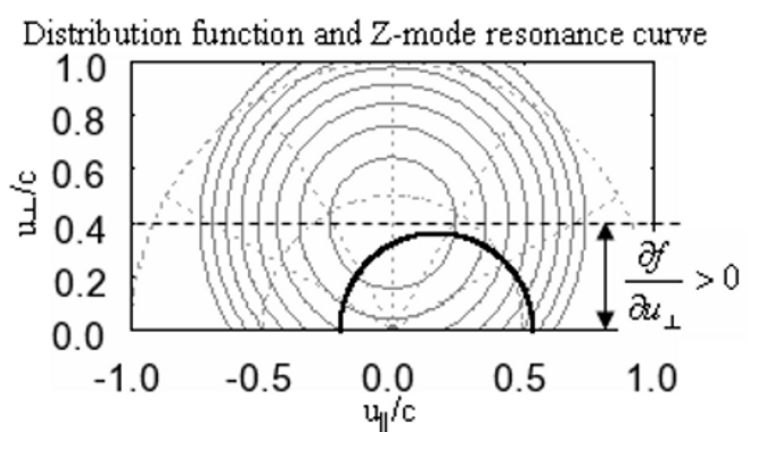

Fig. 4. (Grey) A ring distribution function with $n_{c}=1000 / \mathrm{cc}$, $n_{h}=1 / \mathrm{cc}, u_{\perp 0} / c=0.4$, and $A=0.1$, and (black) the resonance curve of a Z-mode wave in the momentum space where $\omega / \Omega_{e}=2.85$, $\omega_{p} / \Omega_{e}=2.9, \theta=80^{\circ}$, and $n=3$. The interval between contours is $10^{3}$.

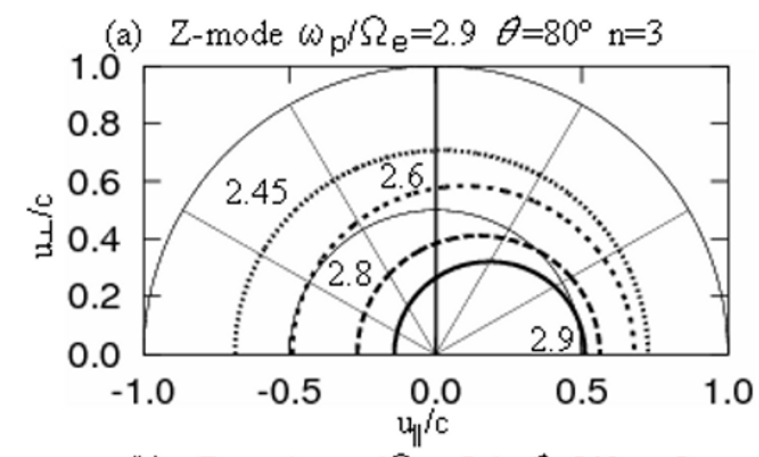

(b) Z-mode $\omega \mathrm{p} / \Omega_{\mathrm{e}}=3.1 \quad \theta=80^{\circ} \mathrm{n}=3$

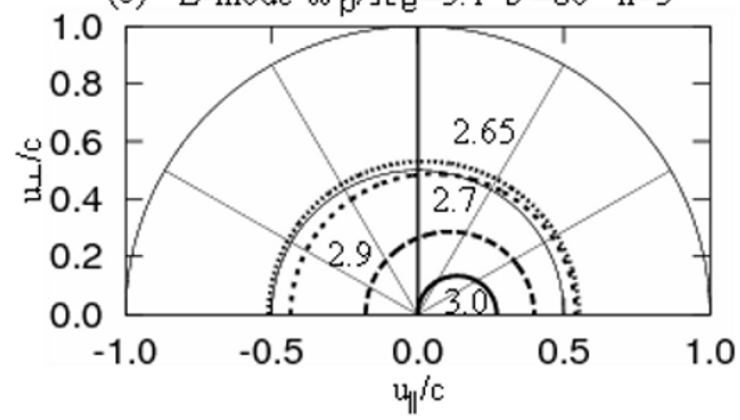

Fig. 5. Resonance curves of Z-mode waves with $\theta=80^{\circ}$ and $n=3$ for (a) $\omega_{p} / \Omega_{e}=2.9$ and (b) 3.1 . The normalized frequencies are shown beside each curve.

Then, the next subject which we should investigate is whether these waves grow up to the observed intensity level during the propagation around the equatorial plasmasphere. The wave energy gain can be estimated as

$$
\exp \left(\int_{0}^{t} \omega_{i} d t\right)=\exp \left(\int_{r_{0}}^{r_{1}} \frac{\omega_{i}}{\mathbf{v}_{g}} \cdot d \mathbf{r}\right) \simeq \exp \left(\sum_{r} \frac{\omega_{i} \Delta r}{v_{g}}\right)
$$

where $\mathbf{v}_{g}$ is the group velocity vector calculated from the Appleton-Hartree's dispersion relation. The ambient plasma density is given by $n_{c}=n_{0} L^{-2.65}$, which is obtained from the observational result by the Akebono/PWS (Ikeda et al., manuscript in preparation), and the ambient magnetic field is assumed to be the dipole magnetic field. Hot plasma is distributed uniformly in space with ring dis- 
(a) $Z$-mode $\omega \mathrm{p} / \Omega_{\mathrm{e}}=2.9 \mathrm{n}=3 \mathrm{u}_{\perp 0}=0.4$

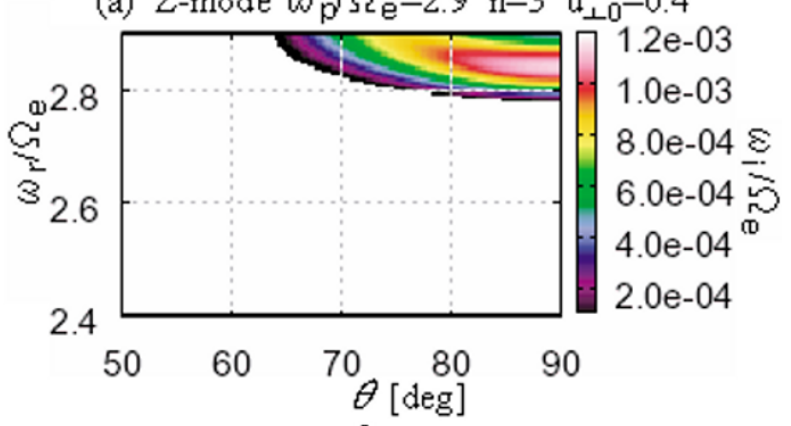

(b) $Z$-mode $\omega \mathrm{p} / \Omega_{\mathrm{e}}=3.1 \mathrm{n}=3 \mathrm{u}_{\perp 0}=0.4$

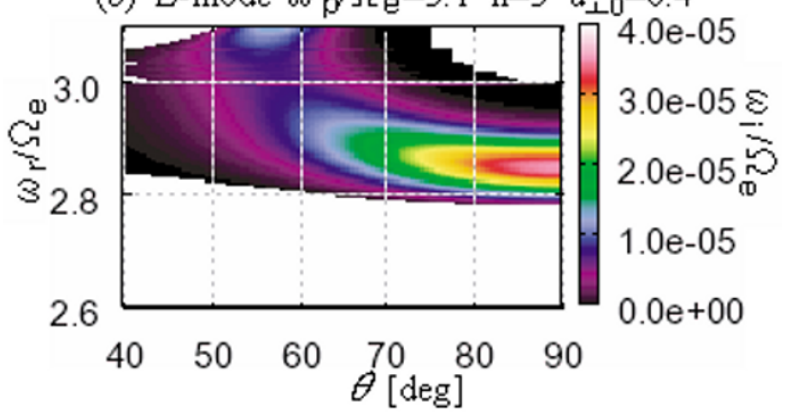

Fig. 6. The growth rate of Z-mode waves as functions of wave normal angles and the real part of the normalized frequency where (a) $\omega_{p} / f_{c}=2.9$ and (b) 3.1. The order of resonance is $n=3$ in the distribution shown in Fig. 4.
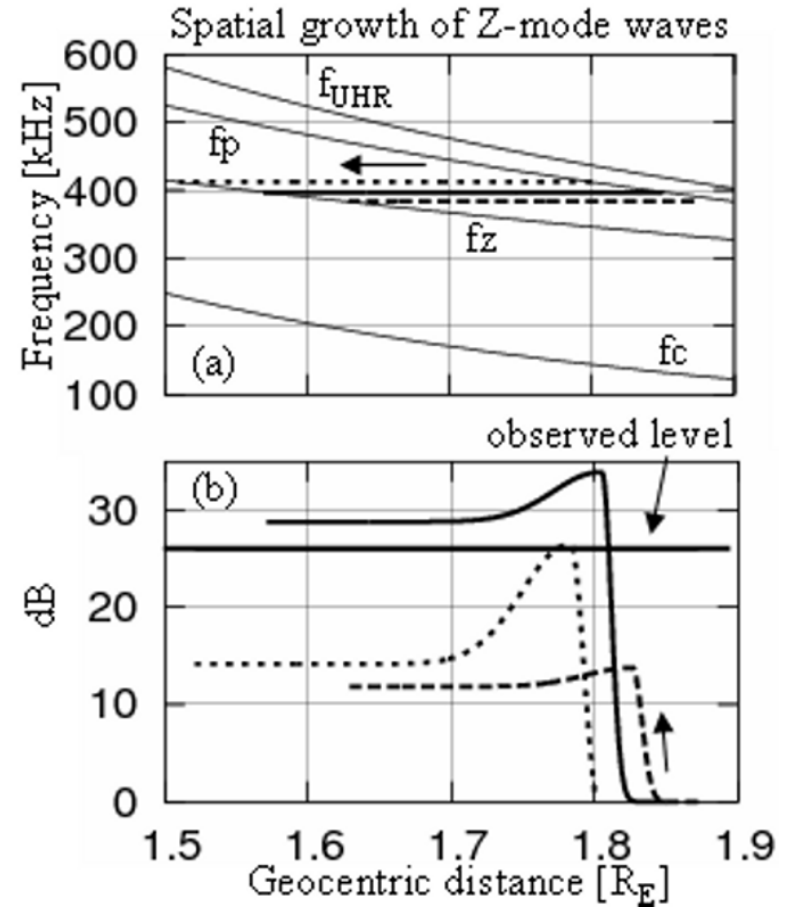

Fig. 7. Intensity variation of Z-mode waves in the equatorial plasmasphere. The panels show (a) The radial variation of the characteristic frequencies and (b) the wave amplitude $[\mathrm{dB}]$ as a function of the geocentric distance. tribution function (4) with $n_{h} / n_{c}=0.001, u_{\perp 0}=0.4$, $A_{\|}=0.1, A_{\perp}=0.1$ within $u_{\perp}<0.2$, and $A=0.2$ within $u_{\perp}>0.2$. In this calculation, the growth rates ware calculated along the ray path of the $\mathrm{Z}$-mode waves.

The result is shown in Fig. 7. Radial variation of $\Omega_{e}, \omega_{z}, \omega_{p}$, and $\omega_{\mathrm{UHR}}$ is shown in the panel (a). Waves start from the region where $\omega=\omega_{p}$ and $\theta=90^{\circ}$, and propagate toward the Earth until these waves reach cut-off at $\omega=\omega_{z}$. The waves effectively grow in a small distance of the first $0.05 R_{E}$ where the wave frequency is just below the ambient plasma frequency. This frequency characteristic is consistent with the observation in Fig. 1. After escaping these regions, amplitudes of these three waves reach the maximum values of $33.8,26.4,13.7 \mathrm{~dB}$, and slightly decrease due to the cyclotron damping by hot plasma because the radii of resonant curves increase. As shown in Fig. 1, the observed Z-mode waves are enhanced about $26 \mathrm{~dB}$ from the background noise level in the equatorial plasmasphere. The energy gains estimated from the present calculation are almost the same level to explain the observed Z-mode waves in Fig. 2. This result suggests that Z-mode waves observed in the equatorial region of the plasmasphere, can be excited directly by the cyclotron-type wave particle interaction.

\subsection{UHR and whistler mode waves}

Intense UHR waves can also be generated by the higherorder cyclotron process under the same distribution function. Figures 8(a) and 9(a) show the resonance curves and corresponding growth rates of UHR waves for the same condition as Fig. 6. The resonance curves are elliptical when UHR waves interact with $\mathrm{keV}$ electrons. Waves with large wave normal angles around $80^{\circ}$ are excited with the maximum growth rate of $\omega_{i}=1.3 \times 10^{-2} \Omega_{e}$ near the UHR frequency. However, frequencies in resonance are strongly limited $\left(\Delta \omega / \Omega_{e} \sim 0.02\right)$. This narrow frequency bandwidth cannot explain the enhanced UHR waves in the whole UHR branch seen in Fig. 1. If the resonance condition for other frequencies in the UHR mode is also satisfied in nearby plasma, the wide spectral bandwidth would be explained by the propagation effect. Otherwise, since UHR and electrostatic waves can interact through the Landau and first order cyclotron resonances, these processes will work more efficiently to excite UHR waves.

The ring-type momentum distribution also has a possibility to produce whistler mode waves through the cyclotron resonance. Figures 8(b) and 9(b) show the resonance curves and growth rate of whistler waves with the same condition in Fig. 6 other than $n=1$ and $\theta=5^{\circ}$. The waves in a frequency range of $0.2<\omega / \Omega_{e}<0.3$ resonate with $\mathrm{keV}$ electrons, and the maximum growth rate reaches $\omega_{i} / \Omega_{e}=4.5 \times 10^{-2}$, which is larger than that of the Zmode waves. This result is consistent with the Akebono observation given in Fig. 1, where whistler waves are observed associated with Z-mode enhancements. The observed frequency range of the lower band whistler waves is coincident with the excited wave in Fig. 6. Therefore, the enhancement of the Z-mode and whistler mode waves supports the existence of energetic electrons with some tens of $\mathrm{keV}$ confined around the geomagnetic equator, and these electrons generate Z-mode and whistler mode waves through the cyclotron interaction. Although whistler waves have growth rates as large as those of UHR or Z-mode waves, these waves are weaker and not as frequently observed as UHR and Z-mode 
(a) UHR mode $\omega \mathrm{p} / \Omega_{\mathrm{e}}=2.9 \quad \theta=80^{\circ} \mathrm{n}=3$

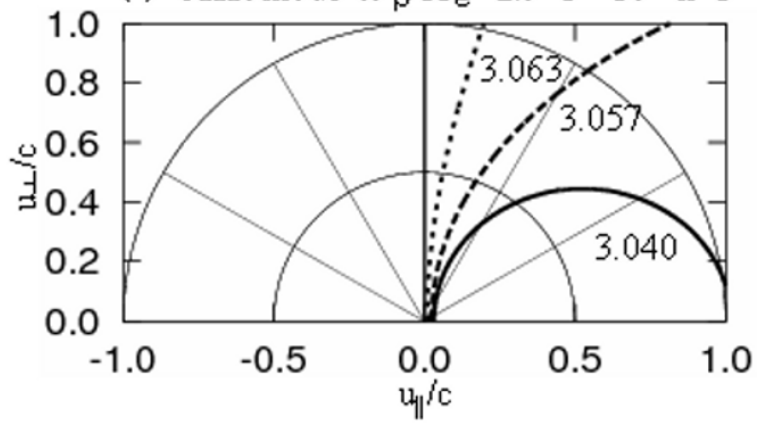

(b) Whistler mode $\omega_{\mathrm{p}} / \Omega_{\mathrm{e}}=2.9 \quad \theta=5^{\circ} \mathrm{n}=1$

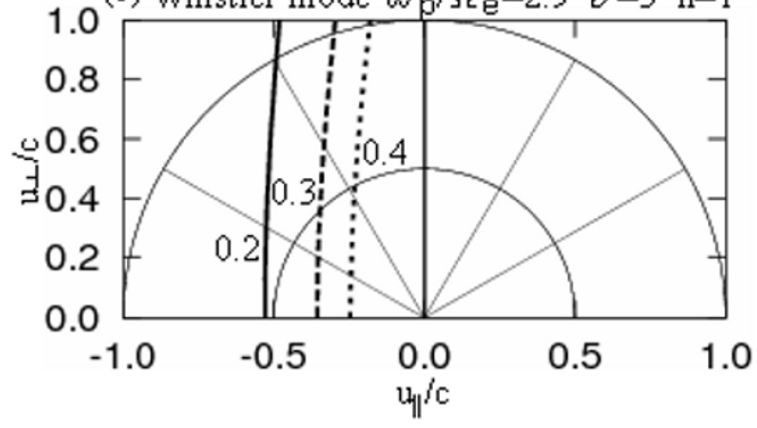

Fig. 8. Resonance curves of (a) UHR and (b) whistler mode waves for the same condition as Fig. 6 other than $n=1$ and $\theta=5^{\circ}$ in panel (b).
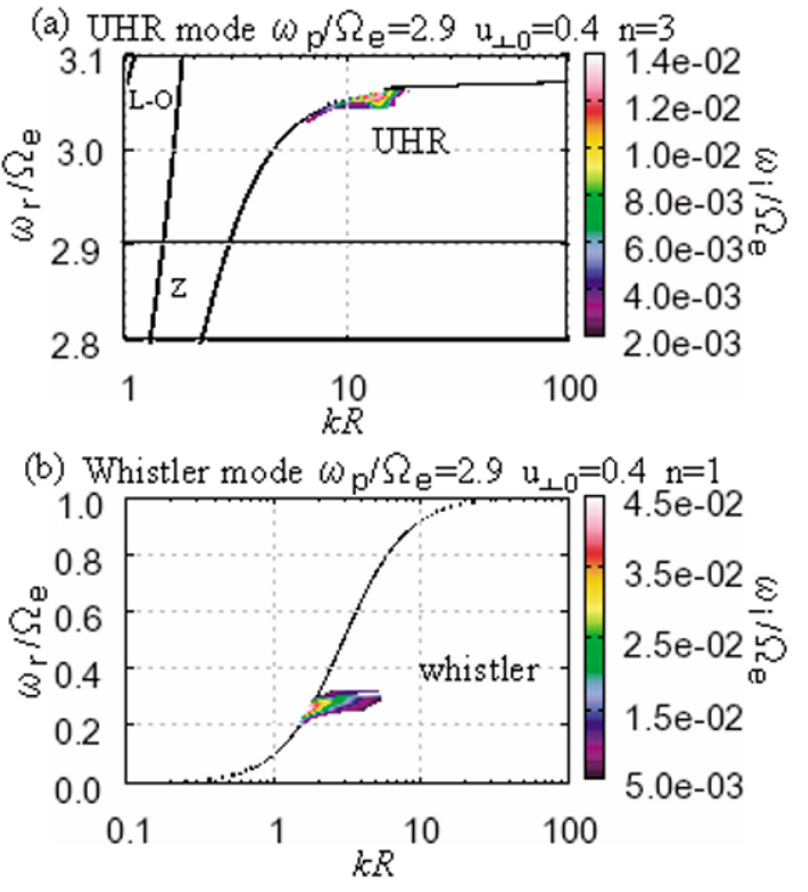

Fig. 9. Growth rates of (a) UHR and (b) whistler mode waves for the same condition as Fig. 6 other than $n=1$ in panel (b). The growth rate is shown in $\omega$ - $k$ diagram. waves. It would be because the excited whistler waves have small wave normal angles, and propagate out of the equatorial region with relatively short time before being amplified enough.

\section{Discussion and Conclusion}

From the Akebono wave measurements, we have shown that Z-mode electromagnetic waves are excited in the equatorial plasmasphere during geomagnetically disturbed periods. Z-mode waves tend to be more intense than UHR waves, indicating that these Z-mode waves are excited directly through a wave-particle interaction rather than the mode conversion from electrostatic waves. A possible candidate of the source population is energetic electrons injected into this region by the intense large-scale electric field during geomagnetic storms. From the trajectory tracing, electrons in the premidnight tail region are accelerated perpendicular to the ambient magnetic field and confined around the geomagnetic equator because of the conservation of the first and second adiabatic invariants. Since the intensity of Z-mode and UHR waves is associated with the development and decay of the ring current, ring current electrons are a probable candidate for the free energy source of these waves after injected into the equatorial plasmasphere.

In order to investigate whether equatorially mirroring electrons excite Z-mode plasma waves directly, linear growth rates of Z-mode waves have been calculated numerically in the high-density backgrounds of the plasmasphere. Z-mode waves interact with tens of $\mathrm{keV}$ electrons with large pitch angles through the higher-order cyclotron resonance. From the calculations of spatial growth rates, $\mathrm{Z}$-mode waves are amplified $33.8 \mathrm{~dB}$. This magnitude is consistent with observations in the equatorial region by the Akebono satellite. The generated Z-mode waves have large wave normal angles, and thus these waves propagate nearly perpendicular to the magnetic field. This explains the confinement of the Z-mode activity around the geomagnetic equator.

Intense UHR waves can also be generated by the equatorially mirroring electrons through the higher-order cyclotron resonance with a large growth rate of $\omega_{i} / \Omega_{e}=$ $1.3 \times 10^{-2}$. However, the frequency bandwidth of $0.02 \Omega_{e}$ is too narrow to explain the enhanced UHR waves in the whole UHR branch. The Landau and first order cyclotron resonances (Harvey, 1968) or propagations from surrounding plasma would contribute to the UHR emissions in the equatorial plasmasphere.

The ring-type momentum distribution of electrons also produces whistler mode waves by the first order resonance. These waves are excited in a frequency range of $0.2<\omega / \Omega_{e}<0.3$ with the maximum growth rate of $\omega_{i} / \Omega_{e}=4.5 \times 10^{-2}$, which is larger than that of the Zmode waves. Generation of whistler waves associated with Z-mode waves is consistent with the Akebono observation. These results suggest that equatorially mirroring energetic electrons exist in the equatorial region, and these electrons excite electromagnetic waves mainly through the cyclotrontype interactions.

This first order resonance is the same process discussed by Kennel and Petscheck (1966) and Wu and Lee (1979). Winglee and Dulk (1986) and Yoon et al. (1998) have considered the higher-order cyclotron maser in a condition of $\omega_{p} / \Omega_{e}>1$ but focused on UHR (slow Z) waves. Our mechanism concerns the generation of fast $\mathrm{Z}$-mode waves 
in high density background, where it has been considered to be difficult to excite electromagnetic waves. Although the growth rate of Z-mode is smaller than the case of the UHRmode, it can explain the amplitude observed by the Akebono satellite. Thus, the higher-order cyclotron resonance is possible to work for the generation of Z-mode electromagnetic waves.

In the present work, since the growth rate calculations are based on the linear theory, quasi-linear and non-linear effects are not taken into account. Distribution functions will be relaxed to a marginal state of stability, and wave growth rates will become smaller than the present results (Yoon et al., 1998). Spatial inhomogeneity of the ambient magnetic field caused by the large amplitude plasma waves will cause the phase trapping of energetic electrons, and the wave frequency will be modulated in the non-linear stage of waveparticle interaction (Katoh and Omura, 2007). However, the wave magnetic field of enhanced Z-mode waves is about $0.1 \mathrm{pT}$ for the observed wave electric field of $10 \mu \mathrm{V} / \mathrm{m}$, which is $10^{-6}$ times smaller than the ambient magnetic field. Thus, the linear approach is still valid to discuss the generation process of enhanced $\mathrm{Z}$-mode waves in the equatorial plasmasphere.

From these results, we can deduce the storm-time evolution of ring current electrons as the origin of plasma waves in the equatorial plasmasphere. Electrons with from $100 \mathrm{eV}$ to $1 \mathrm{keV}$ in the geomagnetic tail region are injected into the inner plasmasphere by storm-time strong ambient electric fields. Since these electrons are perpendicularly heated conserving the first and second adiabatic invariants, energetic electrons are localized in the equatorial plasmasphere with the energy of some tens of keV. These electrons generate UHR, Z-mode and whistler waves mainly by the cyclotrontype interaction process around the geomagnetic equator, drifting around the Earth. Since the amplitude of large-scale electric field depends on geomagnetic activities, the activity of excited plasma waves by these energetic electrons also shows a clear dependence on geomagnetic conditions. This process will explain the origin of the EPWAT phenomena observed by the Akebono satellite.

Acknowledgments. The Akebono satellite was established by the Institute of Space and Astronautical Science (ISAS/JAXA). The SYM-H index was provided by the WDC-C2 for Geomagnetism. This work was supported by the 21st century COE program "Advanced Science and Technology Center for the Dynamic Earth" at the Tohoku University and the Research Fellowship of the Japan Society for the Promotion of Science.

\section{References}

Baldwin, D. E., I. B. Bernstein, and M. P. H. Weenink, Kinetic theory of plasma waves in a magnetic field, in Advances in plasma physics, edited by A. Simon and W. B. Thompson, vol. 3, pp. 1-125, Interscience Publishers, 1969.

Bauer, S. J. and R. G. Stone, Satellite Observations of Radio Noise in the Magnetosphere, Nature, 218, 1145, 1968.

Burke, W. J., N. C. Maynard, M. P. Hagan, R. A. Wolf, G. R. Wilson, L. C. Gentile, M. S. Gussenhoven, C. Y. Huang, T. W. Garner, and F. J. Rich, Electrodynamics of the inner magnetosphere observed in the dusk sector by CRRES and DMSP during the magnetic storm of June 4-6, 1991, J. Geophys. Res., 103, 29,399-29,418, 1998.

Calvert, W., Oblique z-Mode Echoes in the Topside Ionosphere, J. Geophys. Res., 71, 5579, 1966.

Christiansen, P. J., M. P. Gough, G. Martelli, J. J. Bloch, N. Cornilleau,
J. Etcheto, R. Gendrin, and C. Beghin, GEOS-1 observations of electrostatic waves, and their relationship with plasma parameters, Space Sci. Rev., 22, 383-400, 1978.

Eckersley, T. L., Discussion on the Ionosphere, Proc. Roy. Soc., A, 141, $710,1933$.

Gregory, P. C., Radio emission from auroral electrons, Nature, 221, 350, 1969.

Harvey, C. C., Radio emission from geomagnetically trapped particles, Nature, 217, 50-51, 1968.

Horita, R. E. and H. G. James, Two-point studies of fast Z-mode waves with dipoles in the ionosphere, Radio Sci., 39, 4001, 2004.

Jones, D., Latitudinal beaming of planetary radio emissions, Nature, 288, 225-229, 1980.

Katoh, Y. and Y. Omura, Computer simulation of chorus wave generation in the Earth's inner magnetosphere, Geophys. Res. Lett., 34, 3102, 2007.

Kennel, C. F. and H. E. Petscheck, Limit on stably trapped particle fluxes, J. Geophys. Res., 71, 1-28, 1966.

Kutiev, I., K. Oyama, and T. Abe, Analytical representation of the plasmasphere electron temperature distribution based on Akebono data, $J$. Geophys. Res., 107, SMP 24-1, 2002.

Lee, L. C., C. S. Wu, H. P. Freund, D. Dillenburg, and J. Goedert, Excitation of high-frequency waves with mixed polarization by streaming energetic electrons, J. Plasma Phys., 22, 277-288, 1979.

Maynard, N. C., W. J. Burke, E. M. Basinska, G. M. Erickson, W. J. Hughes, H. J. Singer, A. G. Yahnin, D. A. Hardy, and F. S. Mozer, Dynamics of the inner magnetosphere near times of substorm onsets, $J$. Geophys. Res., 101, 7705-7736, 1996.

Melrose, D. B., G. A. Dulk, and R. G. Hewitt, Electron-cyclotron maser emission-Relative growth and damping rates for different modes and harmonics, J. Geophys. Res., 89, 897-904, 1984.

Mishin, E. V. and W. J. Burke, Stormtime coupling of the ring current, plasmasphere, and topside ionosphere: Electromagnetic and plasma disturbances, J. Geophys. Res., 110, 7209, 2005.

Mosier, S., M. Kaiser, and L. Brown, Observations of noise bands associated with the upper hybrid resonance by the Imp 6 radio astronomy experiment, J. Geophys. Res., 10, 1673-1979, 1973.

Nishimura, Y., T. Ono, M. Iizima, A. Shinbori, A. Kumamoto, and H. Oya, Statistical studies of fast and slow Z-mode plasma waves in and beyond the equatorial plasmasphere based on long-term Akebono observations, Earth Planets Space, 58, 343-346, 2006.

Nishimura, Y., A. Shinbori, T. Ono, M. Iizima, and A. Kumamoto, Evolution of ring current and radiation belt particles under the influence of storm-time electric fields, J. Geophys. Res., 2007 (in press).

Oya, H., Studies on Plasma and Plasma Waves in the Plasmasphere and Auroral Particle Acceleration Region, by PWS on board the EXOS-D (Akebono) Satellite, J. Geomag. Geoelectr. Suppl., 43, 369-393, 1991.

Oya, H., A. Morioka, K. Kobayashi, M. Iizima, T. Ono, H. Miyaoka, T. Okada, and T. Obara, Plasma wave observation and sounder experiments (PWS) using the Akebono (EXOS-D) satellite-Instrumentation and initial results including discovery of the high altitude equatorial plasma turbulence, J. Geomag. Geoelectr., 42, 411-442, 1990.

Oya, H., M. Iizima, and A. Morioka, Plasma turbulence disc circulating the equatorial region of the plasmasphere identified by the plasma wave detector (PWS) onboard the Akebono (EXOS-D) satellite, Geophys. Res. Lett., 18, 329-332, 1991.

Roederer, J. G., Dynamics of Geomagnetically Trapped Radiation, in Physics and Chemistry in Space, edited by J. G. Roederer, Denver and J. Zähringer, vol. 2, Springer-Verlag, 1970.

Winglee, R. M. and G. A. Dulk, The electron-cyclotron maser instability as a source of plasma radiation, Ap. J., 307, 808-819, 1986.

$\mathrm{Wu}, \mathrm{C}$. S. and L. C. Lee, A theory of the terrestrial kilometric radiation, Ap. J., 230, 621-626, 1979.

Wygant, J., D. Rowland, H. J. Singer, M. Temerin, F. Mozer, and M. K. Hudson, Experimental evidence on the role of the large spatial scale electric field in creating the ring current, J. Geophys. Res., 103, 29,52729,544, 1998.

Yoon, P. H., A. T. Weatherwax, and T. J. Rosenberg, On the generation of auroral radio emissions at harmonics of the lower ionospheric electron cyclotron frequency: $\mathrm{X}, \mathrm{O}$ and $\mathrm{Z}$ mode maser calculations, J. Geophys. Res., 103, 4071-4078, 1998.

Y. Nishimura (e-mail: yukitoshi@stpp1.geophys.tohoku.ac.jp), T. Ono (e-mail: ono@stpp1.geophys.tohoku.ac.jp), M. Iizima, A. Shinbori, and A. Kumamoto 